\title{
Important comments on KERMA factors and DPA cross-section data in ACE files of JENDL-4.0, JEFF-3.2 and ENDF/B-VII.1
}

\author{
Chikara Konno ${ }^{1, \text { a }}$, Kenichi Tada ${ }^{1}$, Saerom Kwon $^{2}$, Masayuki Ohta ${ }^{2}$, and Satoshi Sato ${ }^{2}$ \\ 1 Japan Atomic Energy Agency, Nuclear Science and Engineering Center, 319-1195 Tokai-mura, Japan \\ 2 National Institutes for Quantum and Radiological Science and Technology, Fusion Energy Research and Development Directorate, \\ 039-3212 Rokkasho-mura, Japan
}

\begin{abstract}
We have studied reasons of differences of KERMA factors and DPA cross-section data among nuclear data libraries. Here the KERMA factors and DPA cross-section data included in the official ACE files of JENDL-4.0, ENDF/B-VII.1 and JEFF-3.2 are examined in more detail. As a result, it is newly found out that the KERMA factors and DPA cross-section data of a lot of nuclei are different among JENDL4.0, ENDF/B-VII.1 and JEFF-3.2 and reasons of the differences are the followings: 1) large secondary particle production yield, 2) no secondary gamma data, 3) secondary gamma data in files $12-15 \mathrm{mt}=3,4$ ) $\mathrm{mt}=103-107$ data without $\mathrm{mt}=600 \mathrm{~s}-800 \mathrm{~s}$ data in file6. The issue 1) is considered to be due to nuclear data, while the issues 2)-4) seem to be due to NJOY. The ACE files of JENDL-4.0, ENDF/B-VII.1 and JEFF3.2 with these problems should be revised after correcting wrong nuclear data and NJOY problems.
\end{abstract}

\section{Introduction}

KERMA factors and DPA cross-section data for calculating nuclear heating and damage in nuclear analyses are stored in ACE and MATXS files [1]. It is known that KERMA factors of a lot of nuclei in the official ACE files are different among nuclear data libraries mainly because of inconsistent energy-balance [2]. We also pointed out that KERMA factors and DPA cross-section data were different among JENDL-4.0 [3], ENDF/B-VII.1 [4], JEFF-3.2 [5] and FENDL-3.0 [6] even for nuclei with consistent energy-balance by the following reasons [7].

i) Nuclear data issue

- Incorrect Q value in nuclear data

- Large gas production cross-section data

ii) NJOY/HEATR module [1] issue

- ${ }^{1} \mathrm{H}$ of ENDF/B-VII.1 (this issue is corrected in NJOY2012.50)

$-\mathrm{mt}=102$ data in file6 [8]

In this paper we study the KERMA factors and DPA crosssection data in the latest official ACE files of JENDL-4.0, ENDF/B-VII.1 and JEFF-3.2 in more detail.

\section{Method}

For all the nuclei (497 nuclei) included in JENDL-4.0, ENDF/B-VII.1 and JEFF-3.2, we extracted the total crosssection data, average heating numbers and damage energy production data from the latest official ACE files.

- JENDL-4.0: AceLibJ40 [9] by Japan Atomic Energy Agency (NJOY99.336 [10] + JENDL-4 patch [11])

- ENDF/B-VII.1: MCNP data [12] by Los Alamos National Laboratory (NJOY99.393)

a e-mail: konno.chikara@jaea.go.jp
- JEFF-3.2: ACE file [5] by OECD/NEA (NJOY99.393+ NEA patch [13]).

We converted the average heating numbers to KERMA factors and the damage energy production data to DPA cross-section data as followings,

$$
\begin{gathered}
K E R M A=H \times \sigma_{t}, \\
D P A=\left(0.8 \times E_{a}\right) /\left(2 \times E_{d}\right)
\end{gathered}
$$

where $H$ is an average heating number, $\alpha_{t}$ is a total cross-section, $E_{a}$ is a damage energy production and $E_{d}$ is an atomic displacement energy. The KERMA factors and DPA cross-section data were plotted for comparison. If necessary, the NJOY2012.50 [1] code was used for processing nuclear data tentatively generated in this study.

\section{Results and discussion}

It is newly found out that KERMA factors and DPA cross-section data of a lot of nuclei are different among JENDL-4.0, ENDF/B-VII.1 and JEFF-3.2. As described in the next sub-sections, reasons for most of the new differences of the KERMA factors and DPA cross-section data among the nuclear data libraries are successfully categorized as the followings,

- large secondary particle production yield,

- no secondary gamma data,

- secondary gamma data in files $12-15 \mathrm{mt}=3$,

$-\mathrm{mt}=103-107$ data without $\mathrm{mt}=600 \mathrm{~s}-800 \mathrm{~s}$ data in file6.

\subsection{Large secondary particle production yield}

The KERMA factors and DPA cross-sections of ${ }^{182} \mathrm{~W}$, ${ }^{183} \mathrm{~W},{ }^{184} \mathrm{~W}$ and ${ }^{186} \mathrm{~W}$ in JEFF-3.2 are extraordinarily

(c) The Authors, published by EDP Sciences. This is an Open Access article distributed under the terms of the Creative Commons Attribution License 4.0 (http://creativecommons.org/licenses/by/4.0/). 


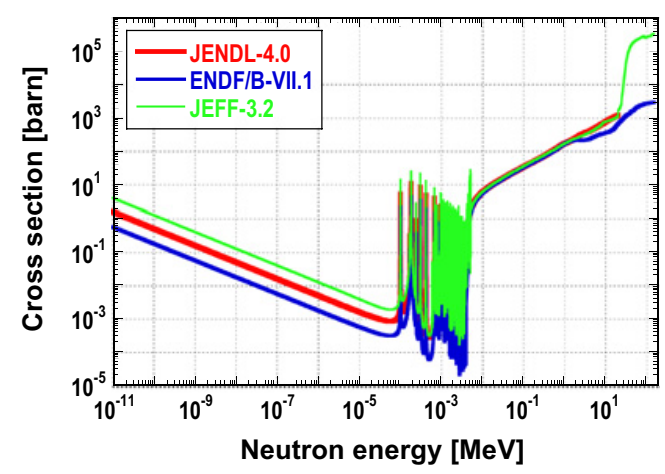

Figure 1. DPA cross-sections of ${ }^{184} \mathrm{~W}$.

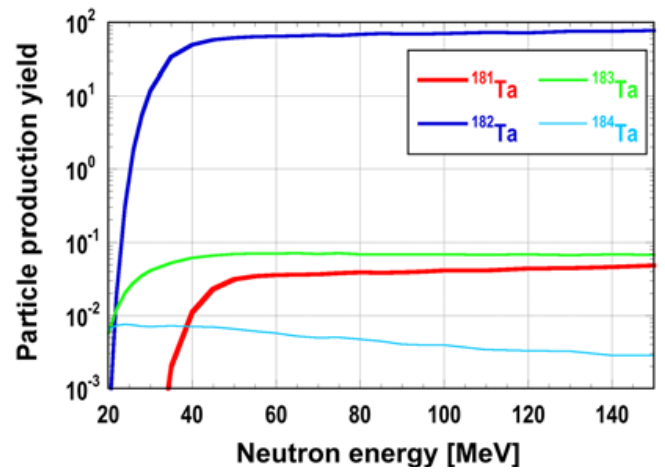

Figure 2. Ta isotope production yields of ${ }^{184} \mathrm{~W}$ in JEFF-3.2.

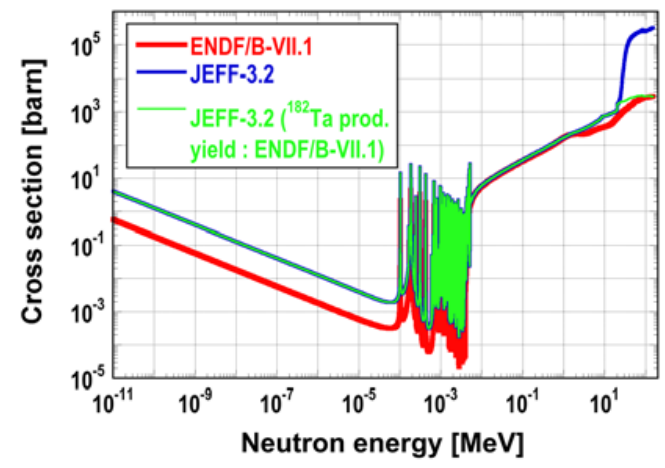

Figure 3. DPA cross-sections of ${ }^{184} \mathrm{~W}$.

large above $20 \mathrm{MeV}$ as shown in Fig. 1. We find that these tungsten data have large particle production yields (e.g., ${ }^{182} \mathrm{Ta}$ in ${ }^{184} \mathrm{~W}$ ) in file6 $\mathrm{mt}=5$ as shown in Fig. 2. In order to confirm that the large particle production yields cause the large KERMA factors and DPA cross-sections, we replace the problematic particle production yield data in ${ }^{182} \mathrm{~W},{ }^{183} \mathrm{~W},{ }^{184} \mathrm{~W}$ and ${ }^{186} \mathrm{~W}$ of JEFF-3.2 with those in ENDF/B-VII.1. Then the large KERMA factors and DPA cross-sections disappear as shown in Fig. 3. The particle production yield data in ${ }^{182} \mathrm{~W},{ }^{183} \mathrm{~W},{ }^{184} \mathrm{~W}$ and ${ }^{186} \mathrm{~W}$ of JEFF-3.2 should be revised.

\subsection{No secondary gamma data}

A lot of nuclei in JENDL-4.0, ENDF/B-VII.1 and JEFF-3.2 have no secondary gamma data. In this case kinematic KERMA factors may be stored in ACE files (e.g., JENDL-4.0 ACE file) as the second best. Kinematic KERMA factors are expected to correspond to energybalance ones in low neutron energy, but it is found that the kinematic KERMA factors are different from the

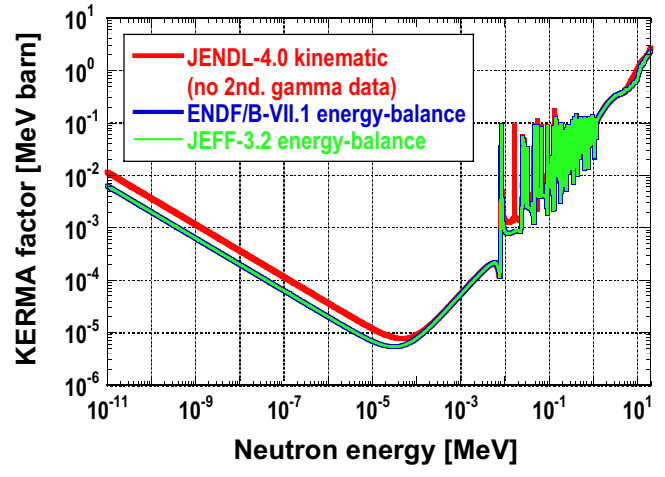

Figure 4. KERMA factors of ${ }^{37} \mathrm{Cl}$.

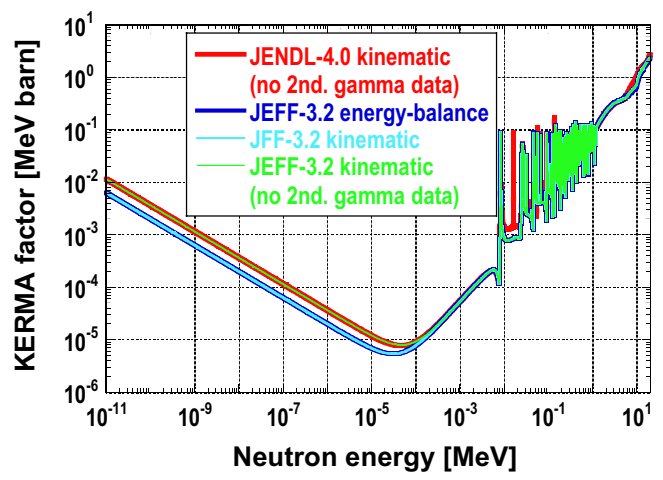

Figure 5. KERMA factors of ${ }^{37} \mathrm{Cl}$.

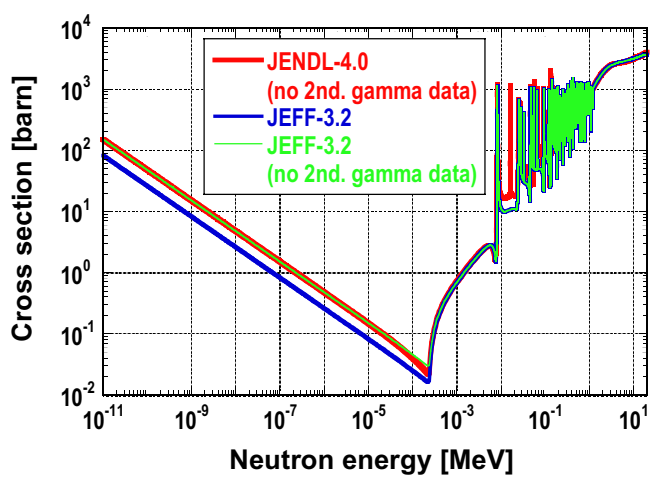

Figure 6. DPA cross-sections of ${ }^{37} \mathrm{Cl}$.

energy-balance KERMA factors with the secondary gamma data in low neutron energy as shown in Fig. 4. Here we consider ${ }^{37} \mathrm{Cl}$. If the secondary gamma data of ${ }^{37} \mathrm{Cl}$ in JEFF-3.2 are deleted, the kinematic KERMA factor of the modified ${ }^{37} \mathrm{Cl}$ is different from the energy-balance KERMA factor of the original ${ }^{37} \mathrm{Cl}$ in JEFF-3.2 and agrees with the kinematic KERMA factor of ${ }^{37} \mathrm{Cl}$ in JENDL-4.0 as shown in Fig. 5. It is noted that the energy-balance and kinematic KERMA factors of the original ${ }^{37} \mathrm{Cl}$ in JEFF3.2 are almost the same. The same issue also occurs in the DPA cross-section data as shown in Fig. 6. It seems that this issue is due to the NJOY code, in other words, NJOY cannot process nuclear data without secondary gamma data adequately.

\subsection{Secondary gamma data in files $12-15 \mathrm{mt}=3$}

JENDL-4.0 has a lot of nuclei with secondary gamma data in files12-15 $\mathrm{mt}=3$, where the similar problem 


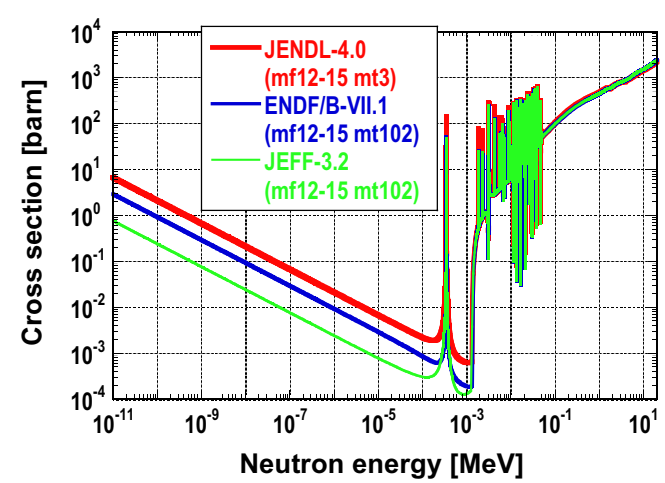

Figure 7. DPA cross-sections of ${ }^{92} \mathrm{Mo}$.

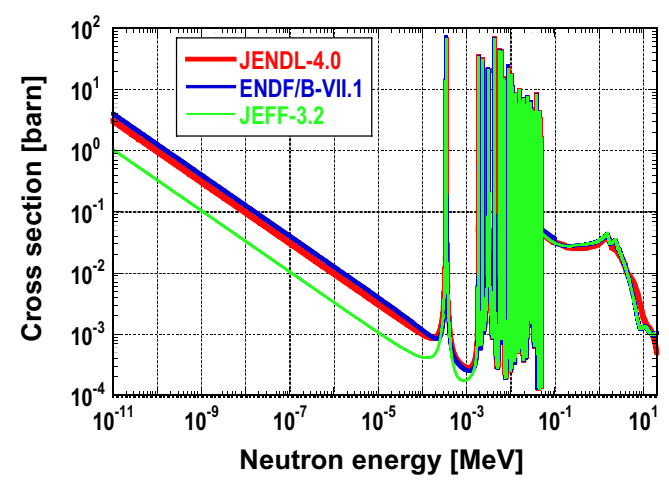

Figure 8. Capture reaction cross-sections of ${ }^{92} \mathrm{Mo}$.

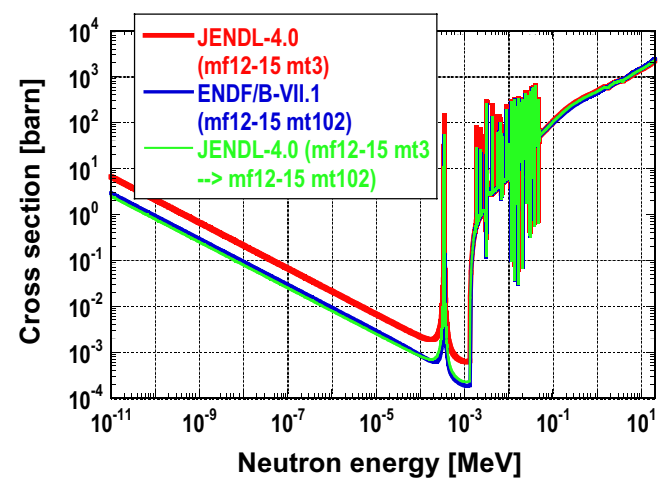

Figure 9. DPA cross-sections of ${ }^{92} \mathrm{Mo}$.

as the previous sub-section is found out. Figure 7 shows a typical example: the DPA cross-sections in ${ }^{92} \mathrm{Mo}$ of the three nuclear data libraries with secondary gamma data. The cross-sections are different below $\sim 1 \mathrm{keV}$. The difference between those of ENDF/B-VII.1 and JEFF-3.2 is considered to come from the difference of the capture reaction cross-section data as shown in Fig. 8. The capture reaction cross-section data of JENDL-4.0 and ENDF/ B-VII.1 are almost the same below $\sim 1 \mathrm{keV}$, but the format of the secondary gamma data for the capture reaction is different between JENDL-4.0 and ENDF/B-VII.1: $\mathrm{mt}=$ 3 for JENDL-4.0 and $\mathrm{mt}=102$ for ENDF/B-VII.1. In order to confirm that the different format of the secondary gamma data for the capture reaction causes the difference of the DPA cross-section data, we convert the files12-15 $\mathrm{mt}=3$ data below $500 \mathrm{keV}$ of ${ }^{92} \mathrm{Mo}$ in JENDL-4.0 to files $12-15 \mathrm{mt}=102$ data. Figure 9 shows that the DPA cross-section of the converted ${ }^{92} \mathrm{Mo}$ in JENDL-4.0 is almost the same as that of ${ }^{92} \mathrm{Mo}$ in ENDF/B-VII.1 below

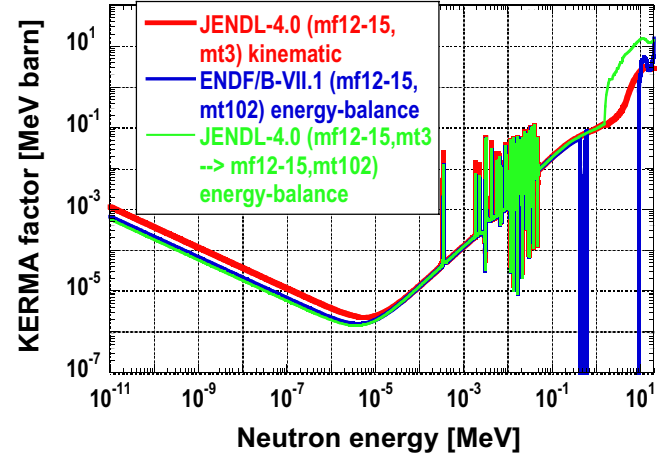

Figure 10. KERMA factors of ${ }^{92}$ Mo. Note that energybalance KERMA factors are incorrect above $400 \mathrm{keV}$ because of inconsistent energy-balance.

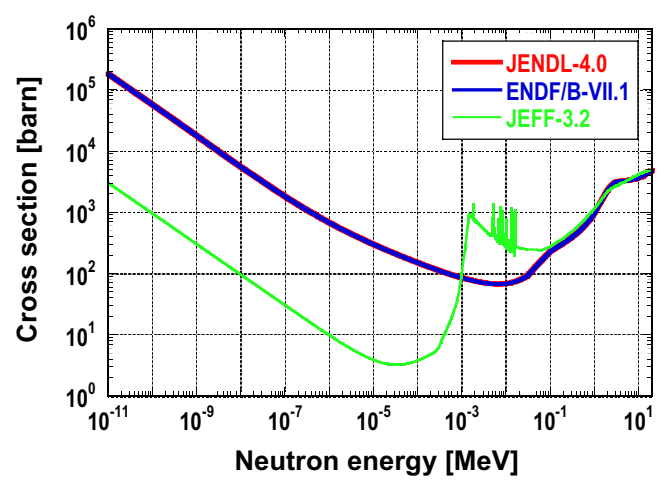

Figure 11. DPA cross-sections of ${ }^{40} \mathrm{~K}$.

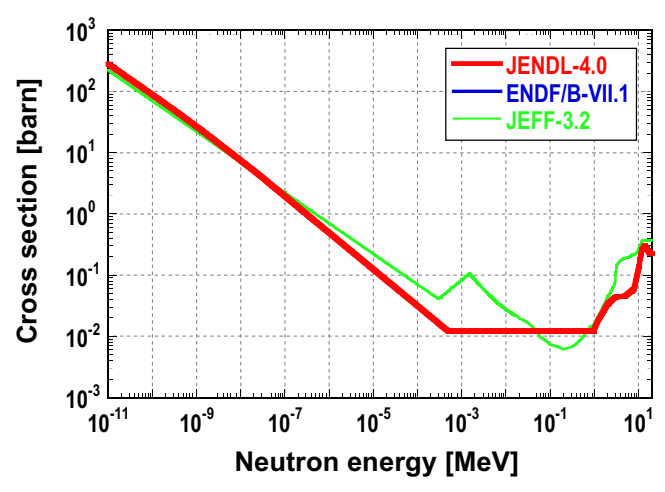

Figure 12. ${ }^{1} \mathrm{H}$ production cross-sections of ${ }^{40} \mathrm{~K}$.

$\sim 1 \mathrm{keV}$. It seems that NJOY cannot process files12-15 $\mathrm{mt}=3$ data adequately.

Most of the nuclear data of nuclei with secondary gamma data in files12-15 $\mathrm{mt}=3$ do not keep energybalance. Then kinematic KERMA factors may be stored in ACE files (e.g., JENDL-4.0 ACE file). The kinematic KERMA factors have the same problem as the DPA crosssection data as shown in Fig. 10. This also seems to be due to NJOY.

\section{4. $\mathrm{mt}=103-107$ data without $\mathrm{mt}=600 \mathrm{~s}-800 \mathrm{~s}$ data in file 6}

The DPA cross-section data of ${ }^{39-41} \mathrm{~K}$ in JEFF-3.2 are much smaller than those in JENDL-4.0 and ENDF/BVII.1 below $\sim 1 \mathrm{keV}$ as shown in Fig. 11. It is noted that the gas production cross-section data of ${ }^{39-41} \mathrm{~K}$ are very large in low neutron energy as shown in Fig. 12 and 


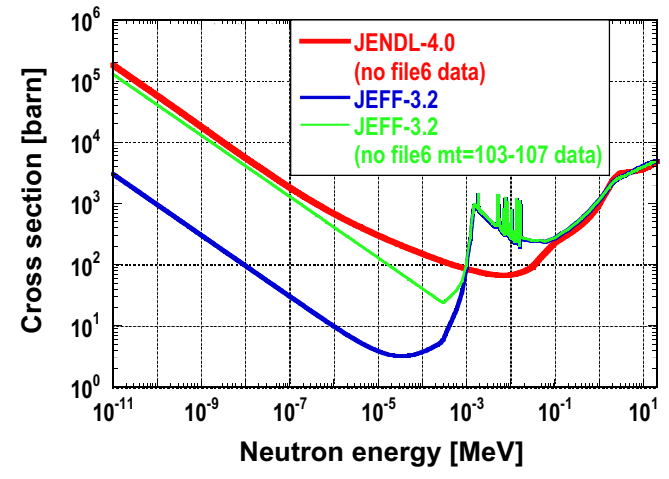

Figure 13. DPA cross-sections of ${ }^{40} \mathrm{~K}$.

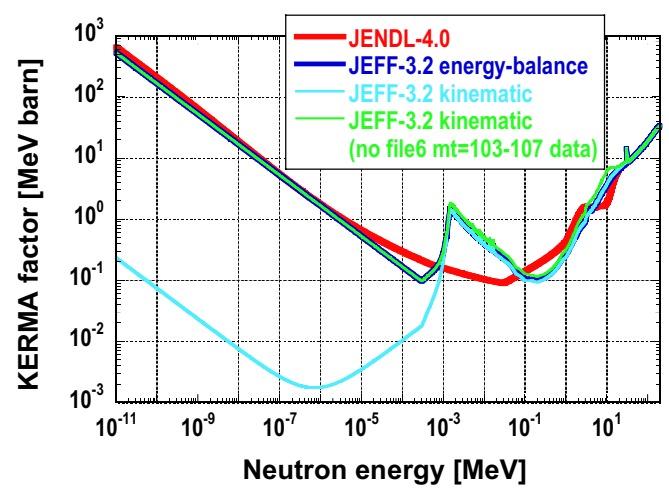

Figure 14. KERMA factors of ${ }^{40} \mathrm{~K}$.

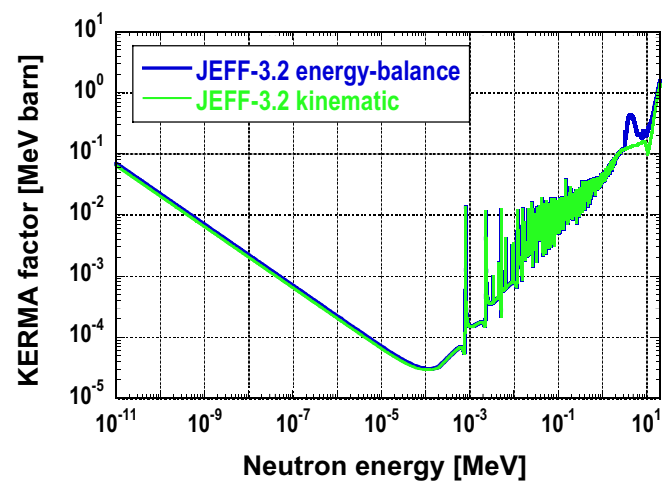

Figure 15. KERMA factors of ${ }^{209} \mathrm{Bi}$. Note that energy-balance KERMA factor is incorrect above a few $\mathrm{MeV}$ because of inconsistent energy-balance.

${ }^{39-41} \mathrm{~K}$ in JEFF-3.2 have the file $6 \mathrm{mt} 103-\mathrm{mt} 107$ data for the gas production reactions, while those in JENDL-4.0 and ENDF/B-VII.1 have no file6 data for the gas production reactions. Here we consider ${ }^{40} \mathrm{~K}$. If the file $6 \mathrm{mt} 103-\mathrm{mt} 107$ data for the gas production reactions of ${ }^{40} \mathrm{~K}$ in JEFF-3.2 are deleted, the DPA cross-section data become almost the same as those in JENDL-4.0 and ENDF/B-VII.1 below
$1 \mathrm{keV}$ as shown in Fig. 13. It seems that NJOY does not process the file $6 \mathrm{mt} 103$-mt107 data adequately.

The energy-balance KERMA factors of ${ }^{39-41} \mathrm{~K}$ in JEFF-3.2 have no problem as shown in Fig. 14, but the kinematic KERMA factors have the same problem as the DPA cross-sections. The ${ }^{209} \mathrm{Bi}$ data in JEFF-3.2 have large gas production cross-section data as ${ }^{39-41} \mathrm{~K}$ in JEFF-3.2, but the secondary charged particle data are stored in file $6 \mathrm{mt}=600 \mathrm{~s}-800 \mathrm{~s}$, not in file $6 \mathrm{mt}=103-107$. In this case the energy-balance and kinematic KERMA factors are the same as shown in Fig. 15. It is considered that NJOY processes file $6 \mathrm{mt}=600 \mathrm{~s}-800 \mathrm{~s}$ data, not file 6 mt103-mt107 data.

\section{Conclusions}

We examined KERMA factors and DPA cross-section data in the official ACE files of JENDL-4.0, ENDF/B-VII.1 and JEFF-3.2 in more detail. It is newly found out that KERMA factors and DPA cross-section data of a lot of nuclei are different among JENDL-4.0, ENDF/B-VII.1 and JEFF-3.2. Reasons for most of the differences of KERMA factors and DPA cross-section data among the nuclear data libraries are successfully categorized as the followings: 1) large secondary particle production yield, 2) no secondary gamma data, 3) secondary gamma data in files12-15 $\mathrm{mt}=3,4) \mathrm{mt}=103-107$ data without $\mathrm{mt}=$ 600 s-800 s data in file6. Incorrect nuclear data and NJOY problems should be revised based on this study and the ACE files with the problems also should be revised.

\section{References}

[1] A.C. Kahler et al., Los Alamos National Laboratory report, LA-UR-12-27079 (2012)

[2] C. Konno et al., Nuclear Data Sheets 118, 450 (2014)

[3] K. Shibata et al., J. Nucl. Sci. Technol. 48, 1 (2011)

[4] M. Chadwick et al., Nuclear Data Sheets 112, 2887 (2011)

[5] https://www.oecd-nea.org/dbforms/data/ eva/evatape s/jeff_32/

[6] R.A. Forrest et al., International Nuclear Data Committee report, INDC(NDS)-0628 (2012)

[7] C. Konno et al., Fusion Eng. Design 109-111, 1649 (2016)

[8] M. Herman and A. Trkov (Ed.), Brookhaven National Laboratory report, BNL-90365-2009 (2009)

[9] http://prodas.jaea.go.jp/?AceLibJ40 in Japanese

[10] http://t2.lanl.gov/nis/codes/njoy99/ index.html

[11] K. Okumura, private communication (2012)

[12] D.L. Conlin et al., Los Alamos National Laboratory report, LA-UR-13-20137 (2013)

[13] O. Cabellos, JEFFDOC-1516 (2014) 